\title{
Delivery of therapeutic radioisotopes using nanoparticle platforms: potential benefit in systemic radiation therapy
}

This article was published in the following Dove Press journal:

Nanotechnology, Science and Applications

2 December 2010

Number of times this article has been viewed

\author{
Longjiang Zhang ${ }^{1,2}$ \\ Hongwei Chen' \\ Liya Wang' \\ Tian Liu Li,4 $^{3,4}$ \\ Julie Yeh' \\ Guangming $\mathrm{Lu}^{2}$ \\ Lily Yang ${ }^{4,5}$ \\ Hui Mao ${ }^{1,4}$ \\ 'Department of Radiology, Emory \\ University School of Medicine, Atlanta, \\ GA, USA; ${ }^{2}$ Department of Medical \\ Imaging, Jinling Hospital, Nanjing \\ University School of Medicine, \\ Nangjing, Jiangsu Province, China; \\ ${ }^{3}$ Department of Radiation Oncology, \\ Emory University School of Medicine, \\ Atlanta, GA, USA; ${ }^{4}$ Emory Winship \\ Cancer Institute, Emory University, \\ Atlanta, GA, USA; ${ }^{5}$ Department of \\ Surgery, Emory University School of \\ Medicine, Atlanta, GA, USA
}

\begin{abstract}
Radiation therapy is an effective cancer treatment option in conjunction with chemotherapy and surgery. Emerging individualized internal and systemic radiation treatment promises significant improvement in efficacy and reduction of normal tissue damage; however, it requires cancer cell targeting platforms for efficient delivery of radiation sources. With recent advances in nanoscience and nanotechnology, there is great interest in developing nanomaterials as multifunctional carriers to deliver therapeutic radioisotopes for tumor targeted radiation therapy, to monitor their delivery and tumor response to the treatment. This paper provides an overview on developing nanoparticles for carrying and delivering therapeutic radioisotopes for systemic radiation treatment. Topics discussed in the review include: selecting nanoparticles and radiotherapy isotopes, strategies for targeting nanoparticles to cancers, together with challenges and potential solutions for the in vivo delivery of nanoparticles. Some examples of using nanoparticle platforms for the delivery of therapeutic radioisotopes in preclinical studies of cancer treatment are also presented.
\end{abstract}

Keywords: cancer, radiation therapy, nanoparticle, radioisotope, delivery

\section{Introduction}

Cancer is one of the leading causes of death worldwide. In 2008, a total of 1,437,180 new cancer cases and 565,650 cancer deaths were estimated in the United States alone. ${ }^{1}$ Despite advances in our understanding of tumor biology, cancer biomarkers, surgical procedures, radio- and chemotherapy, the overall survival rate from cancer has not improved significantly in the past two decades. ${ }^{1}$ Early detection, pathological characterization, and individualized treatments are recognized as important aspects for improving the survival of cancer patients. Many novel approaches, such as imaging for the early detection of molecular events in tumors, comprehensive and personalized treatments, and targeted delivery of therapeutic agents to tumor sites, have been developed by various research groups; and some of these are already in clinical trials or applications for cancer patients. Radiation therapy, in conjunction with chemotherapy and surgery, is an effective cancer treatment option, especially for radiation-sensitive tumors. Radiation therapy utilizes high dose ionizing radiation to kill cancer cells and prevent progression and recurrence of the tumor. In current clinical oncology practices, half of all cancer patients will be treated with radiation therapy either alone or in combination with other treatments.

Traditionally, radiation therapies fall into one of three categories: external radiation, internal radiation and systemic radiation therapy. External radiation therapy delivers high-energy x-rays or electron or proton beams to a tumor from outside the body, often
Department of Radiology, Emory

University School of Medicine, I 364

Clifton Road,Atlanta, GA 30322, USA

$\mathrm{Tel}+\mathrm{I} 4047120357$

Fax +l $4047 / 25948$

Email hmao@emory.edu 
under imaging guidance. Internal radiation therapy (also called brachytherapy) places radiation sources within or near the tumor using minimally invasive procedures. Systemic radiation therapy delivers soluble radioactive substances, either by ingestion, catheter infusion, or intravenous administration of tumor-targeting carriers, such as antibodies or biocompatible materials, which carry selected radioisotopes. Although systemic radiation offers desirable advantages of improved efficacy as well as potentially reducing radiation dosage and side effects, in vivo delivery of radioisotopes with tumor targeted specificity needs to address many challenges that include: 1) the selection of radioisotopes with a proper halflife; 2) a delivery vehicle that can carry an optimal amount of radioisotopes and has favorable pharmacokinetics; 3 ) suitable tumor biomarkers that can be used to direct the delivery vehicle into cancer cells; and 4) specific tumor targeting ligands that are inexpensive to produce and can be readily conjugated to the delivery vehicles. In addition, a multifunctional carrier that not only delivers radioisotopes but also provides imaging capability for tracking and quantifying radioisotopes that have accumulated in the tumor is highly desirable.

Recent advances in nanotechnology have led to the development of novel nanomaterials and integrated nanodevices for cancer detection and screening, in vivo molecular and cellular imaging, ${ }^{2}$ and the delivery of therapeutics such as cancer cell killing radio-isotopes. ${ }^{3,4}$ An increasing number of studies have shown that the selective delivery of therapeutic agents into a tumor mass using nanoparticle platforms may improve the bioavailability of cytotoxic agents and minimize toxicity to normal tissues..$^{5-7}$ In this review, we attempt to provide an overview on developing nanoparticles for carrying and delivering therapeutic radioisotopes for cancer treatment. We will discuss the following topics: selecting nanoparticles and radiotherapy isotopes, strategies for targeting nanoparticles to cancers, and challenges and potential solutions for in vivo delivery of nanoparticles. Some examples of using nanoparticle platforms for the delivery of therapeutic radioisotopes in preclinical studies, detection, and monitoring of therapy will also be presented.

\section{Cancer cell killing radioisotopes and radiation therapy}

Radiation therapy utilizes radiation energy to induce cell death. By directly delivering external radiation beams to a tumor in the patient, external radiation therapy offers a relatively simple and practical approach to cause radiation damage in the tumor. Although the intensity, location and timing for external radiation can be well controlled and modulated, its main disadvantages include: 1) the destruction of normal tissue adjacent to tumors and in the path of the beam;2) the need of high radiation doses for penetrating tissues with a large field or volume; 3 ) prolonged treatment with the requirement of daily hospital visits for 5-6 weeks; and 4) the use of only selected radiation sources due to the technical requirements and limitations of radiation devices and radiation sources (eg, high energy x-rays). Therefore, external radiation treatment may not be applicable to certain cancers and not effective in the improvement of clinical symptoms. ${ }^{8}$ In contrast to external radiation treatment, systemic radiotherapy delivers radiation energy from the radioisotopes that are conjugated to a suitable delivery carrier, such as antibodies, liposome emulsions or nanoparticles with tumor targeting ligands, and transported to the tumor site as illustrated in Figure 1. If the tumor can be accessed from major arteries, the conjugated radioisotopes can be directly infused through a catheter or by intravenous administration. Since tumor targeted and localized delivery of radiation enhances the treatment effect and reduces the toxicity to normal tissue, systemic radiotherapy is considered to be a promising approach for personalized oncology. Although systemic radiotherapy presents major challenges in the design and production of delivery vehicles, it offers great opportunities for the application of novel nanomaterials and nanotechnologies.

\section{Radioisotopes for radiation therapy}

Although many radioisotopes can be used as radiation sources, only a few have been developed and applied in preclinical and in vivo studies. When selecting a candidate for experimental and clinical studies, the advantages and disadvantages of radioisotopes should be evaluated based on their physical and chemical properties, patient and environmental safety, specific requirements for in vivo applications, and technical feasibility. Table 1 summarizes the physical and radiation properties of therapeutic radioisotopes that have been used in previous studies. These radioisotopes can be categorized into three types, ie, $\alpha, \beta$ and auger particles.

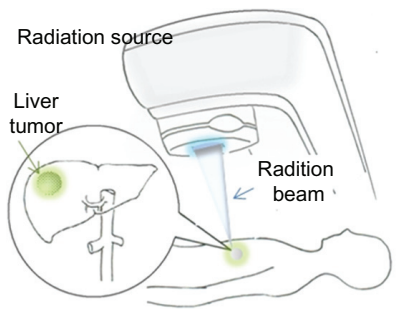

External radiation

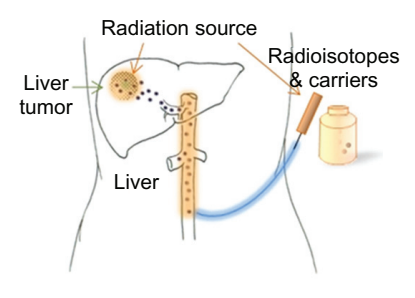

Systemic radiation
Figure I External radiation therapy and systemic radiation therapy. 
Table I Characteristics of some therapeutic radioisotopes

\begin{tabular}{lllll}
\hline Radioisotopes & Particle(s) emitted & Half-life & Particle energy (keV) & Maximum particle range \\
\hline $\begin{array}{llll}\alpha \text {-particle } \\
{ }^{211} \mathrm{At}\end{array}$ & $\alpha$ & 7.2 hours & 6,000 & 0.08 \\
${ }^{225} \mathrm{Ac}$ & $\alpha$ and $\beta$ & 10 days & $6-8,000$ & $0.1 \mathrm{~mm}$ \\
${ }^{212} \mathrm{Bi}$ & $\alpha$ and $\beta$ & 60.6 minutes & 6,000 & $0.09 \mathrm{~mm}$ \\
${ }^{213} \mathrm{Bi}$ & $\alpha$ and $\beta$ & 46 minutes & 6,000 & $<0.1 \mathrm{~mm}$ \\
${ }^{223} \mathrm{Ra}$ & $\alpha$ and $\beta$ & 11.4 days & $6-7,000$ & $<0.1 \mathrm{~mm}$ \\
${ }^{212} \mathrm{~Pb}$ & $\alpha$ and $\beta$ & 10.6 hours & 7,800 & $<0.1 \mathrm{~mm}$ \\
${ }^{149} \mathrm{~Tb}$ & $\alpha$ & 4.2 hours & 4,00 & $<0.1 \mathrm{~mm}$ \\
$\beta-p a r t i c l e$ & & & & $2.0 \mathrm{~mm}$ \\
${ }^{131} \mathrm{I}$ & $\beta$ and $\gamma$ & 193 hours & 610 & $12.0 \mathrm{~mm}$ \\
${ }^{90} \mathrm{Y}$ & $\beta$ & 64 hours & 2,280 & $1.8 \mathrm{~mm}$ \\
${ }^{67} \mathrm{Cu}$ & $\beta$ and $\gamma$ & 62 hours & 577 & $5.0 \mathrm{~mm}$ \\
${ }^{186} \mathrm{Re}$ & $\beta$ and $\gamma$ & 91 hours & 1,080 & $1.5 \mathrm{~mm}$ \\
${ }^{177} \mathrm{Lu}$ & $\beta$ and $\gamma$ & 161 hours & 496 & $2.0 \mathrm{~mm}$ \\
${ }^{64} \mathrm{Cu}$ & $\beta$ & 12.7 hours & 1,670 & $10 \mathrm{~nm}$ \\
Auger-particle & & & & $10 \mathrm{~nm}$ \\
${ }^{67} \mathrm{Ga}$ & Auger, $\gamma$ & 78.3 hours & 90 & $10 \mathrm{~nm}$ \\
${ }^{1231}$ & Auger, $\gamma$ & 13.3 hours & 159 & 27 \\
${ }^{125}$ & Auger, $\gamma$ & 60.5 days & & \\
\hline
\end{tabular}

Abbreviation: keV, kilo electron volts.

\section{$\alpha$-emitters}

More than 100 different radioisotopes emit $\alpha$-particles; however, most of them decay too quickly to be considered for therapeutic use. Only a few $\alpha$-emitters, including actinium-225 $\left({ }^{225} \mathrm{Ac}\right)$, astatine-211 $\left({ }^{211} \mathrm{At}\right)$, bismuth-213 $\left({ }^{213} \mathrm{Bi}\right)$ and bismuth-212 $\left({ }^{212} \mathrm{Bi}\right)$, have therapeutic potential and these have been investigated in animal models or humans. $\alpha$-particles are positively charged helium nuclei with a higher energy $(5,000-8,000 \mathrm{keV})$ and shorter range $(50-80 \mu \mathrm{m})$ than $\beta$-particles. Correspondingly, $\alpha$-particles have a higher linear energy transfer (LET), approximately $100 \mathrm{keV} / \mu \mathrm{m}$, than $\beta$-particles. LET refers to the average radiation energy deposited in tissue per unit length of track $(\mathrm{keV} / \mu \mathrm{m})$. Cell death occurs only when $\alpha$-particles traverse the cell nucleus rather than going directly to the cytoplasm. Owing to these features, $\alpha$-particles are better suited to the treatment of microscopic and small-volume tumors, or residual tumors. In these instances, $\alpha$-particles are more efficient and specific in causing tumor cell death than $\beta$-particles, as shown in many studies. ${ }^{9-11}$ Earlier studies using the single-cell model showed that one cell-surface decay of the $\alpha$-emitter ${ }^{211}$ At resulted in the same degree of cell killing as approximately 1,000 cell-surface decays of the $\beta$-emitter ${ }^{90} \mathrm{Y} .{ }^{12}$ The therapeutic effect of several $\alpha$-emitters has been investigated for decades. Recently, $\alpha$-emitter therapy has received renewed interest, especially for the treatment of microscopic, small tumors or minimal residual tumors ${ }^{13,14}$ in a variety of cancer types, including leukemia, lymphoma, glioma, melanoma, and peritoneal carcinomatosis. However, poor radionuclide supply, complicated methodologies for calculating the radiation dosimetry and the need for relevant data relating to normal organ toxicity limit the applications of $\alpha$-emitter radioisotopes and impede the development of targeted $\alpha$-emitters. ${ }^{15}$

\section{$\beta$-emitters}

Radioisotopes that function as $\beta$-emitters are widely used in radioimmunotherapy trials. Commonly used $\beta$-emitter radioisotopes are iodine-131 $\left({ }^{131} \mathrm{I}\right)$, yttrium-90 $\left({ }^{90} \mathrm{Y}\right)$; copper-67 $\left({ }^{67} \mathrm{Cu}\right)$, rhenium-186 $\left({ }^{186} \mathrm{Re}\right)$, lutetium-177 $\left({ }^{177} \mathrm{Lu}\right)$, and copper-64 $\left({ }^{64} \mathrm{Cu}\right) .{ }^{131} \mathrm{I}$ and ${ }^{90} \mathrm{Y}$ are the most popular candidates since these two isotopes are readily available and inexpensive. ${ }^{131} \mathrm{I}$ has a long half-life ( 8 days) and also provides $\gamma$-emissions that can be used in imaging for tracking and quantifying the radioisotope in vivo. Furthermore, relatively straight forward chemical modifications can be applied to crosslink ${ }^{131}$ I chelates to the proteins, allowing for the labeling of tumor targeting antibodies with radioisotopes. ${ }^{16,17}$ However, ${ }^{131}$ I has some drawbacks, such as rapid degradation and a short retention time in the tumor. ${ }^{13}$ Additionally, the high energy $\gamma$-emission presents some safety concerns to patients and the environment. ${ }^{90} \mathrm{Y}$ has fewer environmental radiation restrictions than ${ }^{131} \mathrm{I}$ because of its pure $\beta$-emitter nature, higher energy and low-range $(12 \mathrm{~mm})$, and a longer residence time in the tumor, making it more suitable for the irradiation of large tumors that require a higher radiation dose and a stable link between radioisotopes and the tumor targeting antibody. ${ }^{13}$ 
$\beta$-particles have lower LET and longer radiation ranges than $\alpha$-particles. Because of their long radiation range (several millimeters), $\beta$-particles can destroy tumor cells through the "crossfire effect," even though the radioimmunoconjugate is not directly bound to the cells. Therefore, they are particularly useful in overcoming treatment resistance. However, longrange $\beta$-particles can produce nonspecific cytotoxic effects by destroying surrounding normal cells. Thus, $\beta$-particles are considered to be most suitable for the treatment of bulky or large volume tumors. ${ }^{18}$

\section{Auger-emitters}

An auger is a high LET ( $4-26 \mathrm{keV} / \mu \mathrm{m})$, low energy $(\leq 1.6 \mathrm{keV})$, and short- range $(\leq 150 \mathrm{~nm})$ electron derived from inner-shell electron transitioning. During the decay of these radioisotopes, the vacancy formed in the $\mathrm{K}$ shell as a consequence of electron capture or internal conversion is rapidly filled by electrons dropping in from higher shells, resulting in a cascade of atomic electron transitions and emitting a characteristic X-ray photon or an auger. Auger emitters, such as gallium-67 $\left({ }^{67} \mathrm{Ga}\right)$, iodine$123\left({ }^{123} \mathrm{I}\right)$ and iodine-125 $\left({ }^{125} \mathrm{I}\right)$, have also been used for cancer radiotherapy. Auger emitters deposit high LET over extremely short distances and are therefore most effective when the decay occurs in the nucleus and less so when the decay occurs in the cytoplasm. The dimensions of many mammalian cell nucleus components, such as 30-nm chromatin fiber, fall in the range of the auger emitter $(<150 \mathrm{~nm})$; therefore, auger emitters are more damaging to those cellular structures. As a result, the use of auger emitters has been relatively restricted because of the extreme toxicity of such radioisotopes. ${ }^{19}$

The selection of radioisotopes for cancer therapy should take into account the specific cancer types, characteristics of the tumor, toxicity and safety of radioisotopes, availability and production of radioisotopes, and the chemistry that is involved in assembling the radioisotopes to the delivery carriers. It has been shown that a combination of radioisotopes with different energies can be more beneficial than using a single radioisotope. For example, a previous study showed that the combination of a high energy and long tissue range radioisotope with a medium energy and shorter tissue range radioisotope is able to destroy both large-volume tumors and micrometastases. ${ }^{13}$

\section{Antibodies conjugated radioisotopes for tumor targeted radiation therapy}

Most conventional anticancer agents do not greatly differentiate between cancerous and normal cells, leading to systemic toxicity and adverse effects, which limits the maximum allowable dose of the agent. The use of monoclonal antibodies $(\mathrm{mAb})$ interacting with cancer cell surface markers is a simple tumor targeting strategy that can lead to a considerable enhancement of treatment efficacy. ${ }^{20}$ Small, high affinity antibody fragments, such as single chain antibodies and affibodies, which are expressed as recombinant proteins in prokaryotic cells, have the additional advantage of cost-effective production of their targeting ligands. ${ }^{21} \mathrm{mAbs}$ or $\mathrm{mAb}$ fragments as ligands for the delivery of radioisotopes, as internal radiation sources, have been investigated extensively. ${ }^{22-24}$ The method of using $\mathrm{mAbs}$ conjugated with radioisotopes for internal or systemic radiation treatment is also known as radioimmunotherapy.

However, there are several limitations in using antibodyconjugated radioisotopes for the delivery of radiation therapy. Firstly, mAbs may bind to cell surface markers that are expressed at a low level in normal tissues, causing potential systemic toxicity. Secondly, mAbs have only a few sites available for conjugating radioisotopes. Therefore, delivery of a large dose of therapeutic radioisotopes may require a larger amount of antibodies. Thirdly, the use of mAbs presents potentially unwanted immune responses. Additionally, antibodies may be susceptible to protease degradation. These limitations may be addressed using nanomaterial based delivery systems.

\section{Biocompatible nanoparticles The chemistry and physical properties of nanomaterials}

Nanoparticles are typically colloidal materials that can be fabricated with a variety of compositions and morphologies using special techniques and chemistries. Nanomaterials currently used in biomedical applications include fluorescent $\mathrm{CdSe}$ nanoparticles known as quantum dots (QDs), photosensitive gold nanoparticles, magnetic nanoparticles, as well as polymer based polymeric nanoparticles and nanoscale liposomes. Nanoparticles, especially metallic and metal oxide nanoparticles, in the "mesoscopic" size range of 5-100 $\mathrm{nm}$ in diameter often exhibit unique chemical and physical properties that are not possessed by their bulk or molecular counterparts. ${ }^{25}$ For example, QDs made from CdSe exhibit photoluminescence with a controllable wavelength ranging from the visible to near infrared depending on their size. Colloidal gold nanoparticles exhibit unique surface plasmon resonance (SPR) properties derived from the interaction of electromagnetic waves with the electrons in the conduction band. ${ }^{26}$ Magnetic nanoparticles, such as iron III oxide $\left(\mathrm{Fe}_{3} \mathrm{O}_{4}\right)$, are superparamagnetic and exhibit high magnetization and yet no residual magnetization in the absence of an externally applied magnetic field. ${ }^{27}$ Both 
the chemical properties and reactivity of the nanoparticles are controlled by the surface chemistries offered by functionalized polymer coatings or blocks, which are also important to the stability and biocompatibility of the nanoparticles, interactions between particles, biomolecules and cells, in addition to tissue distributions of the nanoparticles.

Nanoparticles provide a large surface area and various types of functional groups that allow for chemical reactions taking place on the nanoparticle surface and to assemble or load bioactive ligands or small molecular agents. Diverse nanomaterials with unique properties have been found in various biomedical applications, including in vitro or in vivo imaging, separation and purification of cells or biomolecules, and delivery of therapeutic agents.

\section{Biocompatibilities and functionalization of nanoparticles}

Well designed polymers can be used as coatings to stabilize metallic or metal oxide nanoparticles from aggregating and precipitating in physiological conditions while maintaining the desired physical properties. These polymers should also provide biocompatibilities that include minimizing toxicity from metallic nanoparticles, modulating interactions between nanoparticles and biomolecules, cells and tissues, together with altering the secretion and biodistribution of the nanoparticles. Coating polymers can be functionalized with reactive functional groups, such as $-\mathrm{COOH},-\mathrm{NH}_{2}$ and $-\mathrm{SH}$, which are prepared for the conjugation of the tumor targeting ligands or other agents as illustrated in Figure 2. For carrying and delivering therapeutic radioisotopes, coating polymers with reactive functional groups allows for covalent cross-linking or non-covalently incorporating chelates of radioisotopes.

There are a variety of polymers and their derivatives, such as dextran, polyethylene glycol (PEG), and dendrimer, developed for ensuring the biocompatibility and functionalization of nanoparticles. ${ }^{28,29}$ For instance, Zhang and co-workers modified the surface of iron oxide nanoparticles with trifluoroethyl ester-terminal-PEG-silane, which was then converted to an
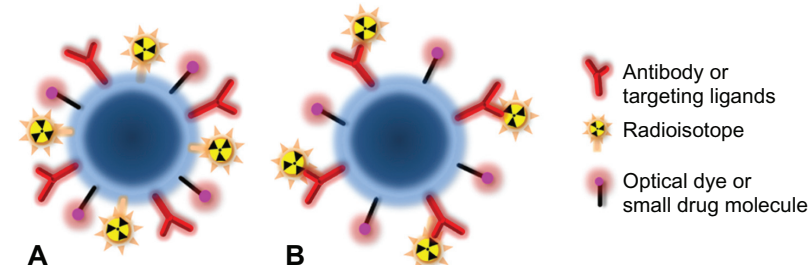

Figure 2 Multifunctional nanoparticle platforms for tumor targeting, imaging, and delivery of drugs and/or radioisotopes.

Notes: Radioisotopes can be conjugated directly on the nanoparticle surface $(\mathbf{A})$ or on the tumor targeting antibodies (B). amine-terminated PEG. ${ }^{30}$ The terminal amine groups were used for the conjugation of Cy5.5, a near infrared (NIR) optical probe, and chlorotoxin, a targeting peptide for glioma tumors. In vitro MRI and confocal fluorescence microscopy showed a strong preferential uptake of the multimodal nanoparticles by glioma cells compared to the control nanoparticles and noncancerous cells, indicating the cancer targeting abilities of the probes for gliomas. To reduce nonspecific uptake of nanoparticles by normal tissues and extend the blood circulation time of nanoparticles to allow particle accumulation at the target site, polymer coatings must be specifically designed to meet such applications. The physical characteristics of polymer coated nanoparticles affect their in vivo performance. Surface morphology, overall particle size and surface charge are all considered important factors that determine toxicity and biodistribution. The overall particle size must be small enough to evade uptake by reticuloendothelial system (RES) but large enough to avoid renal clearance, leaving a window of between 5.5 and $200 \mathrm{~nm} .{ }^{31}$ However, it has also been demonstrated that for particles smaller than $40 \mathrm{~nm}$ in diameter, both the biodistribution and the half-life of nanoparticles are determined by the coating material rather than the mean size. ${ }^{32}$ Nanoparticles with different coating materials usually have different surface charges, which plays a critical role in blood half-lives. It has been demonstrated that both positively and strong negatively charged particles tend to nonspecifically stick to cells. ${ }^{33,34}$ Thus, it is generally considered that nanoparticles with a neutral surface charge are recommended to extend circulation times. Additionally, nanoparticles with neutral surfaces also show a high stability for resisting protein binding and provide steric hindrance for preventing aggregation after in vivo administration. ${ }^{31}$ Recently, new coating materials from zwitterionic polymers have been developed to provide a biocompatible surface with both positive and negative charges, which exhibit high resistance to nonspecific protein adsorption. ${ }^{35}$ In addition, new polymer coatings exhibit high resistance to nonspecific protein binding and uptake by macrophages in the normal liver and spleen. ${ }^{36,37}$ Combining PEG with a crosslinkable polysiloxane coating, Chen and colleagues developed an antibiofouling copolymer PEO- $b$-P $\gamma$ MPS for coating nanocrystals. ${ }^{38}$ This new copolymer made hydrophobic iron oxide nanoparticles mono-disperse in physiological conditions with great stability. Furthermore, this amphiphilic blocked coating polymer can be functionalized with reactive amine groups on the particle surface, making it readily available for the conjugation of tumor targeting ligands and therapeutic agents such as radioisotope chelates. Furthermore, these composite nanoparticles showed a reduced nonspecific uptake. 


\section{Advantages of using nanoparticles to deliver therapeutic agents}

One of the major obstacles in the delivery of therapeutic agents, especially small molecular agents such as radioisotope chelates, is rapid elimination of the agents and their widespread distributions into normal organs and tissues. One common solution to this problem is the administration of the agent in a large quantity, which is not cost-effective and often results in undesirable toxicity. Nanoparticles with proper biocompatible polymer coatings potentially provide better platforms for carrying therapeutic agents and subsequently delivering the agents to the tumor. There are several advantages of using nanoparticles to deliver therapeutic radioisotopes, for instance: 1) nanoparticles have prolonged blood retention time, ranging from 30 minutes to more than 24 hours, depending on the morphology and size of the particle, coating materials, and compositions of nanoparticle conjugates; 2) nanoparticle carriers targeting cancer cells are capable of enhancing local accumulation of the nanoparticles with therapeutic agents, leading to high tumor retention times and concentrations of the agents; 3 ) nanoparticles can load more drug molecules or radioisotopes on a single particle than conventional carriers, sometimes more than one type of therapeutic agent or radioisotope; 4) internalization of receptor targeted nanoparticles leads to the uptake of large amounts of radioisotopes into the target cells, resulting in effective killing of tumor cells with a relatively low level of receptor-expression; and 5) the unique chemical and physical properties of nanoparticles, such as magnetization and photosensitizing, provide additional capabilities and functions for improving delivery of the radioisotopes (for example the external application of a magnetic field) and monitoring the response to radiotherapy. With the controlled formulation of nanoparticles and carefully planned drug carrying strategies, nanoparticle platforms may offer the appropriate pharmacokinetics to enable optimal delivery of therapeutic radioisotopes for cancer treatment.

Biodegradable nanoparticles can be employed to gradually release drugs. For example, therapeutic agents can be interspersed within a poly(DL-lactide-co-glycolide) (PLGA) matrix. As the biocompatible polymer is degraded, the therapeutic agents are released in a sustained manner. ${ }^{39}$ Additionally, particles such as micelles are useful in drug delivery since their hydrophobic centers allow for drug encapsulation while their hydrophilic surfaces allow for ease in transport. ${ }^{40}$ Alternatively, the particle surface allows for modifications since it provides functional groups the opportunity to conjugate small molecular agents. Radioisotopes are typically loaded onto the nanoparticles using the conjugation strategy shown in Figure 3.
Nanoparticle delivery platforms often provide a multi-functional capacity that enables loading multiple moieties, such as targeting ligands and therapeutic agents, using multiple reaction steps. This is particularly important to the tumor targeted delivery of radioisotopes in vivo. It has been reported that nanoparticles consisting of streptavidin that linked three biotinylated components: the antiHer2 antibody trastuzumab, the tat peptide, and the ${ }^{111}$ In-labeled antiRIa messenger RNA antisense morpholino (MORF) oligomer, produce significant radiation-induced antisensemediated cytotoxicity of tumor cells in vitro. ${ }^{41}$

\section{Strategies for targeting nanoparticles to cancers}

Two mechanisms, passive and active targeting, are typically used in targeting nanoparticles to tumors. In passive targeting, nanoparticles and payloads reach the tumor through rich, chaotic, and highly permeable tumor vasculature and are accumulated and subsequently remain in the tumor due to its lack of lymphatic drainage. In active targeting, nanoparticles are engineered to target specific biomarker molecules that are unique and over populated in a tumor or cancer cell surface. Extensive reviews and discussions on the mechanisms of targeting nanoparticles to tumors are beyond the scope of this review and can be found elsewhere. ${ }^{3,42-44}$ Active targeting nanoparticles carrying therapeutic agents, such as radioisotopes,
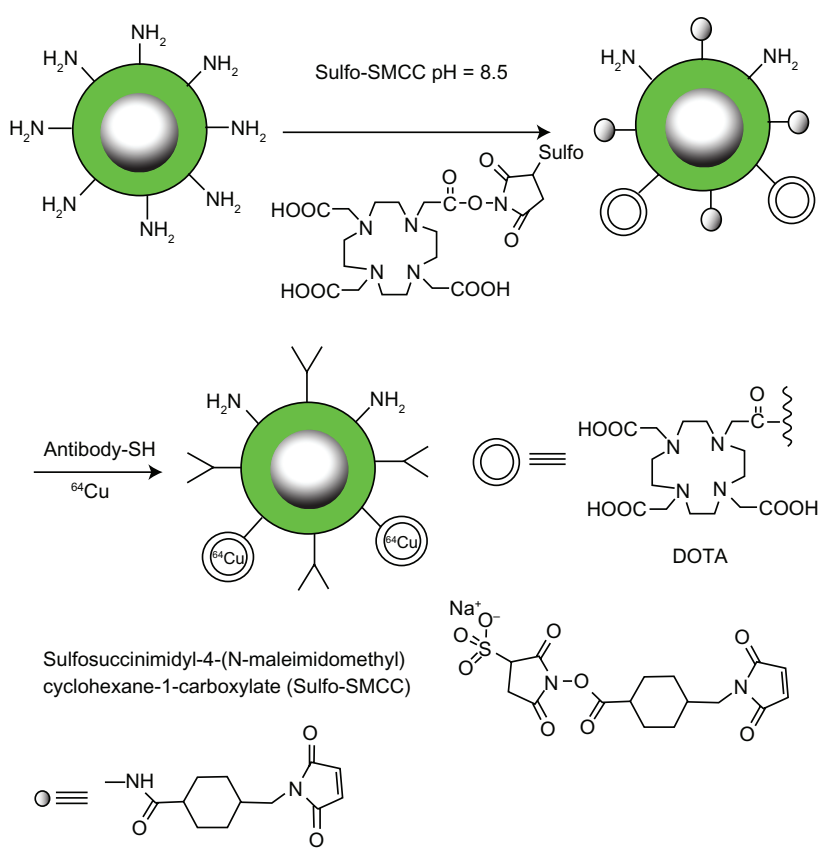

Figure $3 \mathrm{~A}$ scheme for conjugating radioisotope chelates to surface functionalized nanoparticles coated with amphiphilic polysiloxane block polymers containing active $-\mathrm{NH}_{2}$ groups. 
to tumors are the current research focus and the subject of intensive investigations. Differences in the expression of cellular receptors between normal and tumor cells represent a great opportunity for targeting nanoparticles to cancer cells. For engineering tumor targeted nanoparticles, different ligands, such as antibodies, peptides, and small molecules targeting the related receptors that are highly expressed in tumor cells, are usually conjugated to the surface coating polymer of nanoparticles. There have been some reports of in vivo tumor targeting with various targeting ligand/nanoparticle combinations, such as folic acid-modified dendrimers $\left(\approx 9 \% \mathrm{ID} \mathrm{g}^{-1}\right.$; ID $\mathrm{g}^{-1}=$ injected dose per gram tissue) ${ }^{45}$ synthetic small-moleculemodified iron oxide $(\mathrm{IO})$ nanoparticles $\left(\approx 4 \% \mathrm{ID} \mathrm{g}^{-1}\right),{ }^{46}$ polyethylene glycol- coated (PEGylated) arginine - glycine - aspartic acid (RGD) peptide-modified carbon nanotubes (10\%-15\% $\left.\mathrm{ID} \mathrm{g}^{-1}\right),{ }^{47}$ and PEGylated single-chain variable fragment $(\mathrm{ScFv})$ antibody-modified gold nanoparticles $\left(\approx 8 \% \mathrm{ID} \mathrm{g}^{-1}\right) .{ }^{26}$ Tumortargeted nanoparticles as potential carrier vectors of therapeutic agents are believed to be the next promising platform for nanobiotechnology.

Using antibodies as tumor targeting ligands for magnetic or photosensitive nanoparticles has been extensively studied in vitro and in vivo in the area of cancer imaging. Conjugates composed of nanoparticles and antibodies were found to maintain the properties of both the antibody and the nanoparticle. Conjugation of magnetic iron oxide nanoparticles with Herceptin, a well-known antibody against the HER2/neu receptor which is over-expressed in breast cancer cells, showed in vivo cancer targeting and imaging of HER2/neu with a high sensitivity, which enables the magnetic resonance imaging (MRI) detection of tumors as small as $50 \mathrm{mg}$. The smallest parts of the antibody, the variable regions or so called ScFv, are among the most frequently used ligands. Because antibody fragments lack the $\mathrm{Fc}$ domain that binds to $\mathrm{Fc}$ receptors on phagocytic cells, particulates derived with mAb fragments have increased circulation times in the blood compared to particulates derived with whole mAbs. ${ }^{48}$

In contrast to whole mAbs and antibody fragments, small molecule ligands typically can be readily obtained from chemical syntheses in a large quantity, which may be an important factor in translating novel methods into clinical practices. Small peptide ligands, such as tumor integrin $\alpha_{\mathrm{v}} \beta_{3}$ targeted high-affinity Arg-Gly-Asp (RGD) ligand which has a higher binding affinity in its conformationally constrained cyclic form than in its linear form, have been extensively investigated for their in vitro and in vivo applications of delivering tumor targeted nanoparticles carrying imaging and therapeutic agents. The RGD peptide, which has a higher binding affinity in cyclic conformation than its linear form, is able to bind to $\alpha_{\mathrm{v}} \beta_{3}$ or $\alpha_{\mathrm{v}} \beta_{5}$ integrins that are highly expressed in angiogenic tumor endothelial cells and subpopulations of tumor cells. It is likely that RGD-targeted nanoparticles can act on tumor endothelial cells and produce anti-angiogenesis effect. ${ }^{49,50}$ The folate receptor (FR) is an attractive molecular target for tumor targeting because it is over expressed by several types of tumor cells (eg, ovarian, colorectal, breast, nasopharyngeal carcinomas), however, it has limited expression in most normal tissues. ${ }^{51-52}$ FR-mediated tumor delivery of various agents, such as therapeutic drugs and gene products as well as imaging agents with radionuclides or nanoparticles for imaging, have been reported. ${ }^{53-55}$ Folic acids targeting cancer cells over expressing folate receptors have been covalently conjugated to $66 \mathrm{~nm}$ liposomes via spacers of various lengths to target the liposomes to $\mathrm{kB}$ cells with a high level of folate receptor expression. The binding of folate-PEG liposomes to $\mathrm{kB}$ cells can be competitively inhibited by excess free folate or by antiserum against the folate receptor, demonstrating that the interaction is mediated by the cell surface folate-binding protein. These folate-PEG liposomes show potential for delivering large quantities of low molecular weight compounds into folate receptor-bearing cells.

\section{Delivery of tumor targeted therapeutic radioisotopes with nanoparticles}

With surface functionalized nanoparticles and a range of surface chemistries for the conjugation of peptide ligands and antibody moieties, tumor targeting antibodies cross-linked with therapeutic radioisotopes or radioisotope chelates are readily conjugated to nanoparticles. Integrin targeted nanoparticle carrying radioisotopes have demonstrated its effect on tumor vasculatures. In the study by Li and colleagues, integrin antagonist (IA) 4-[2-(3,4,5,6-tetrahydro pyrimidin-2-ylamino) ethoxy]-benzoyl-2-(5)- aminoethylsulfonylamino- $\beta$-alanine, which binds to the integrin $\alpha_{\mathrm{v}} \beta_{3}$, and a monoclonal antibody against murine Flk-1, were used to target nanoparticles radiolabeled with ${ }^{90} \mathrm{Y} \cdot{ }^{22} \mathrm{~A}$ single treatment with IA-nanopartcile- ${ }^{90} \mathrm{Y}$ caused significant tumor growth delay in murine tumor models K1735-M2 (melanoma) and CT-26 (colon adenocarcinoma), compared to untreated tumors, as well as tumors treated with anti-Flk-1 mAb, anti-Flk-1 mAb-NP, and conventional radioimmunotherapy with ${ }^{90}$ Y-labeled anti-Flk mAb.

However, selection of nanoparticle carriers and radioisotopes should be based on which nanoparticle carrier can improve the pharmacokinetics and enhance the delivery of 
therapeutic agents, their therapeutic effects and the additional functionalities offered by nanoparticle carriers as demonstrated in several previous studies are discussed here.

\section{Slow clearance and prolonged blood circulation with nanoparticle carriers}

One major benefit of using nanoparticles to deliver therapeutic radioisotopes for radiation treatment is that the large size and high molecular weight of nanoparticle carriers cause the nanoparticle-radioisotope conjugates to be cleared from the body slowly. Nanoparticle-radioisotope conjugates may have longer blood circulation time than radioisotopes alone or mAb-radioisotope conjugates. The slow clearance and long blood retention time of nanoparticle carriers can be demonstrated by our study on the delivery time courses of optically sensitive ATF-Cy5.5 conjugates and ATF-Cy5.5iron oxide nanoparticle conjugates in mice bearing orthotopic pancreatic xenography tumors (Figure 4). ATF-Cy5.5 conjugates were found to accumulate in the tumor in less than two hours and subsequently be secreted through the kidney in 7 hours after intravenous administration of the agent. However, ATF-Cy5.5- iron oxide nanoparticle conjugates started to accumulate in the tumor after 4 hours and the accumulation continued, peaking at 48 hours. Slow clearance and long blood retention time are critically important to the active targeting of tumors because cell surface marker targeted agents need time to reach inside the tumor and high and effective concentration of agents in the blood facilitates delivery of the agents. The unique pharmacokinetics of nanoparticle carriers has strong implications in optimizing internal radiation sources with proper consideration of the half time of the radioisotopes, radiation dosages, and treatment regime.

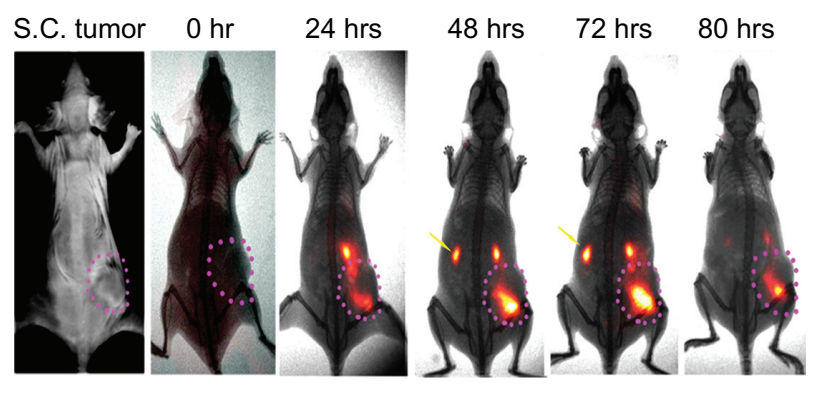

Figure 4 Time dependent accumulation of urokinase plasminogen activator receptor (UPAR) targeted-ATF-nanoparticle-NIR conjugates using amino terminal fragment (ATF) peptide of uPA as uPAR targeting ligands.

Notes: Receptor targeted nanoparticles with optical dye Cy5.5 conjugated on the surface slowly accumulated in a subcutaneously implanted 4TI human breast cancer xenograft as indicated by gradually increase of NIR signal at the site of the tumor with signal intensity peaking between $48-72$ hours after intravenous administration of the nanoparticle complex.
Rossin and colleagues developed ${ }^{64} \mathrm{Cu}$-radiolabeled folate-conjugated shell cross-linked nanoparticles as candidate agents to shuttle radionuclides and drugs into tumors over expressing the folate receptor. ${ }^{56}$ They reported that ${ }^{64} \mathrm{Cu}$-radiolabeled folate-conjugated shell cross-linked nanoparticles exhibited a long circulation time in blood and were able to passively accumulate in tumors. Because ${ }^{64} \mathrm{Cu}$ is also a radioisotope capable of positron emission tomography (PET), ${ }^{64} \mathrm{Cu}$-radiolabeled folate-conjugated shell cross-linked nanoparticles are a class of promising radioisotope agents for imaging and treating early-stage solid tumors.

\section{Improve local delivery and tumor retention time of radioisotopes with magnetic nanoparticles}

Systemic radiation therapy consists of a focused radiation source that remains at the tumor site over a period time. Magnetic nanoparticles, such as biocompatible superparamagnetic iron oxide nanoparticles with core sizes of 10-50 nm, suit this purpose well. Applying an external magnetic field to magnetic nanoparticle-based radioisotopes delivered to tumors may enhance their therapeutic effect and decrease the adverse effect of systemic radiotherapy. ${ }^{57}$ A static magnetic field can be applied after the radioisotopes have localized in the tumors, constraining these magnetic nanoparticles to helical paths, which would result in substantially confining the emitted particles within the tumor's boundaries, thus increasing the radiation dose to the tumor. ${ }^{58}$ Chen and colleagues ${ }^{24}$ developed a radioimmunoconjugate- ${ }^{131} \mathrm{I}$ - anti-vascular endothelial growth factor (VEGF) monoclonal antibody (Sc-7269) crosslinked to dextran coated magnetic iron oxide nanoparticles and investigated their therapeutic effect and safety in nude mice bearing human liver cancer. While ${ }^{131} \mathrm{I}$ - anti-VEGF monoclonal antibody (Sc-7269) provides tumor targeting and a radiation source, the use of magnetic nanoparticles was intended to improve the delivery of the radioisotopes to the tumor and tumor retention time of the radiation source under the force of an external magnetic field. Their results suggested that the radioimmunotherapy of intratumoral injection of ${ }^{131} \mathrm{I}$ - Sc-7269 - nanoparticles is safe and efficient for the treatment of liver cancer.

\section{Radiation treatment combined with hyperthermia induced by nanoparticles}

Local hyperthermia at the site of the tumor has been shown to be an effective cancer treatment because increasing the temperature in living tissues to $42-46^{\circ} \mathrm{C}$ leads to cell death. ${ }^{59}$ Heat can be induced in cancer cells through the external 
application of an alternating magnetic field (AMF) and tumor targeted nanoparticles. Nanoparticle-based hyperthermia has been demonstrated to be a potential method for treating cancer. ${ }^{60}$ Furthermore, application of the external AMF can activate nanoparticles carrying radioisotopes to preferentially be retained in the microenvironment of the tumor tissue at higher concentrations than in the surrounding normal tissue. In an earlier study by DeNardo and colleagues, investigators evaluated the pharmacokinetics, tumor uptake, and the therapeutic effect of ${ }^{111}$ In-chimeric L6 (ChL6) monoclonal antibody (mAb)-linked iron oxide nanoparticles in a thymic mice bearing human breast cancer (HBT 3477) xenografts when inductively heating these ${ }^{111} \mathrm{In}-\mathrm{ChL} 6 \mathrm{mAb}$ linked iron oxide nanoparticles with an externally applied AMF. ${ }^{23}$ They found significant therapeutic responses from AMF/ bioprobe therapy with up to an eight times longer mean time to quintuple tumor volume with the therapy compared with no treatment. Their data suggested that mAb-conjugated nanoparticles, when given intravenously, escaped into the extravascular space and bonded to cancer cell membrane antigens, so that ${ }^{111} \mathrm{In}-\mathrm{ChL} 6 \mathrm{mAb}$ linked iron oxide nanoparticles could be used in concert with an externally applied AMF to deliver thermoablative cancer therapy.

Natarajan and colleagues developed a new class of radioconjugated nanoparticles ( ${ }^{111} \mathrm{In}$-DOTA-di-scFv-NP) for the imaging and therapy of anti-MUC-1-expressing cancers. ${ }^{61}$ They showed the binding of radioconjugated nanoparticles to cancer cells was time dependent with increased binding over time. They also compared the ability of $20 \mathrm{~nm}, 30 \mathrm{~nm}$, and $100 \mathrm{~nm}$ nanoparticles to conjugate ${ }^{111} \mathrm{In}-\mathrm{mAb}$ and found that $100 \mathrm{~nm}$ nanoparticles could be conjugated to ${ }^{111} \mathrm{In}-\mathrm{mAb}$ so that the resulting radioimmunonanoparticles (RINP) had characteristics suitable for AMF therapy. Although $100 \mathrm{~nm}$ RINP targeted tumors less efficiently than $20 \mathrm{~nm}$ RINP, their heating capacity is typically 6 times greater, suggesting the $100 \mathrm{~nm}$ RINP could still deliver better therapy with AMF. ${ }^{62}$

\section{Improving boron neutron capture therapy with nanoparticles}

Boron neutron capture therapy (BNCT) is based on a nuclear reaction in which a neutron of appropriate energy reacts with the stable ${ }^{10} \mathrm{~B}$ isotope, and a high-energy $\alpha$-particle is released in addition to a recoiling ${ }^{7} \mathrm{Li}$ nucleus. The energy of the released $\alpha$-particle provides the main therapeutic effect and is deposited in the tissue within $10 \mu \mathrm{m}$ of the site of neutron capture. This localized therapy is designed to minimize toxicities. Because the recoil range of the emitted lithium and helium particles in tissue is 5 and $7 \mu \mathrm{m}$, respectively, which is comparable to the size of a single cell, the cellular internalization and subcellular localization of the boron carrier with respect to the cell nucleus is a major determinant of the therapeutic efficacy for this approach. ${ }^{63}$ BNCT has been effectively used in treating prostate and breast cancers in clinical oncology. Using monoclonal antibodies serving both as the targeting ligand and the delivery carrier is challenging for this therapy. Recently, various nanoparticle approaches, such as dendrimers, liposomes, cationic acrylamide copolymers ${ }^{64}$ and single-walled carbon nanotubes, ${ }^{65}$ have been developed and tested to deliver boron isotopes that target tumors. For example, boron carbide nanoparticles are studied as a system for T cell-guided boron neutron capture therapy. ${ }^{66}$ Nanoparticles were produced by a method of ball milling in various atmospheres using commercially available boron carbide. In vitro thermal neutron irradiation of B16 melanoma cells incubated with sub-100 nm nanoparticles $\left(381.5 \mathrm{mg} / \mathrm{g}{ }^{10} \mathrm{~B}\right)$ induced complete cell death, while the nanoparticles alone induced no toxicity.

\section{Challenges and future directions of nanoparticle delivery of therapeutic radioisotopes Improving systemic delivery of nanoparticles}

One of the major challenges in the application of nanoparticles in vivo is the nonspecific uptake of nanoparticles by the liver and spleen in the RES when using systemic delivery. For tumor targeted delivery of therapeutic agents, such as radioisotopes, the trapping followed by a rapid clearance of nanoparticles by the RES effectively reduces the tumor targeting efficiency of nanoparticles and potentially causes undesirable organ and normal tissue damage after agents accumulate in the liver and spleen. Therefore, minimizing and evading RES uptake and improving blood retention time of nanoparticles are active areas of research and development in the field. Several strategies, which are mainly focused on optimizing surface coating polymers, have been investigated. PEGylation and reducing surface charge of nanoparticle coatings may significantly reduce the RES uptake and improve the tumor targeting of nanoparticles as demonstrated by many groups. ${ }^{67-69}$ Antibiofouling and increased renal excretion rather than RES clearance were observed in nanoparticles with surface modified silica containing coating polymers. ${ }^{70,71}$

\section{Multifunctional nanoparticles}

In recent years, there has been a tremendous interest in developing multifunctional nanomaterials for cancer detection, treatment, treatment monitoring and image-guided 
interventions. ${ }^{4,48,70}$ For example, theranostic nanoparticles are capable of simultaneous imaging and therapy. Nanoparticles carrying cancer cell killing radioisotopes represent an ideal theranostic system with the radioisotope providing both therapeutic and imaging modalities or combining its therapeutic capability with the imaging capability of nanoparticle carriers. In particular, radioisotopes ${ }^{111} \mathrm{In}$ or ${ }^{64} \mathrm{Cu}$, which emit gamma rays during their decay, have been most frequently used as the imaging probes for PET. Other intrinsic properties of nanoparticles may offer additional functions to further improve tumor specific radiation treatment with the combination of other therapeutics; for example, the ability to induce hyperthermia and increase tumor retention time with magnetic iron oxide or photosensitizing and photoacoustics from gold nanoparticles. Trimodal imaging probes have also been designed by adding another imaging modality to dual MRI and optical imaging probes. The representative multimodal imaging probe is iron oxide nanoparticles coupled with fluorescent organic dyes. The iron oxide nanoparticles chemically crosslinked with dextran have been widely used for in vivo as well as in vitro MRI because of their biocompatibility, small hydrodynamic size, and stable hydrophilic polymer coating. Nahrendorf et al ${ }^{72}$ developed multimodal imaging probes composed of magnetic iron oxide nanoparticles conjugated with arginyl peptides, near infrared (NIR) fluorescent indocyanine dye, Cy5.5, and DTPA to chelate PET tracer ${ }^{64} \mathrm{Cu}$, resulting in a PET, MRI, and optically detectable imaging agent. In another example, RGD peptide was used for the targeted delivery of nanoparticles into integrin $\alpha_{\mathrm{v}} \beta_{3}$-positive tumors. Both small-animal PET and $\mathrm{T}_{2}$-weighted MRI showed integrin-specific delivery of conjugated RGD-polyaspartic acid-iron oxide nanoparticles and prominent RES uptake. ${ }^{70}$ The bifunctional imaging approach may allow for earlier tumor detection with a high degree of accuracy and provide further insight into the molecular mechanisms of cancer.

\section{Improving detection and follow-up of therapy effects}

In addition to its therapy function, nanoconstructs that are capable of reporting responses to therapeutics, especially in the real time monitoring of tumor changes at the molecular level post-treatment, are highly desirable. Imaging techniques, especially clinically feasible modalities in MRI, single photon emission computed tomography (SPECT) and PET, play important roles in the evaluation of the therapeutic effects of radiation therapy. For example, in a recent study by Jacene and colleagues fluorine-18 fluorodeoxyglucose ( $\left.{ }^{18} \mathrm{~F}-\mathrm{FDG}\right)$ PET/CT was used for monitoring the response of nonHodgkin's lymphoma to radioimmunotherapy. ${ }^{73}$ They found
${ }^{18} \mathrm{~F}$-FDG uptake in tumors typically drops significantly after radioimmunotherapy in non-Hodgkin's lymphoma. A continued decline in tumor maximum standardized uptake values between 12 and 24 weeks without additional therapy can occur, suggesting a need for delayed-response assessment. In patients who progress after radioimmunotherapy, new sites of disease, rather than recurrence or progression at previous disease sites, is commonly detected. Large declines in ${ }^{18} \mathrm{~F}$-FDG uptake tend to be seen in those with the longest progression-free survival. Another recent study investigated the efficacy and side-effects of ${ }^{90} \mathrm{Y}$ ibritumomab tiuxetan $\left(\right.$ Zevalin $\left.^{\circledR}\right)$ in patients with earlystage extranodal indolent lymphoma of the ocular adnexa with ${ }^{111}$ In ibritumomab tiuxetan imaging. ${ }^{74}$ All patients had ${ }^{111}$ In ibritumomab tiuxetan imaging to confirm expected biodistribution of ${ }^{90} \mathrm{Y}$-Zevalin during the radiation therapy. Investigators concluded rituximab followed by ${ }^{90} \mathrm{Y}$ ibritumomab tiuxetan was an effective and safe frontline treatment for early-stage extranodal indolent B-cell lymphoma of the ocular adnexa.

\section{Conclusion}

With the advances in nanoscience and nanotechnology, many nanomaterials including nanoparticles made of metal oxide, dendrimers, liposomes, polymers, and micelles can be used as carriers to deliver chemical, pharmaceutical agents and/ or therapeutic radioisotopes into tumors. Conjugating these nanoparticles with therapeutic isotopes and targeted antibodies can enhance therapeutic efficacy and decrease the toxicity and destruction of surrounding normal tissue by radioisotopes. In experimental and clinical studies, proper radioisotope selection that is specific to a particular tumor type and delivery mechanism should be considered when planning in vivo nanoparticle delivery. For example, $\alpha$-particles should be used when micrometastatic diseases or minimal residual tumors are treated, while $\beta$-particle isotopes are recommended for bulky tumors. The half-life of radioisotopes should be considered when using an intravenous system of delivering nanoparticleradioisotope conjugates to the tumor, given the different pharmacokinetics in nanoparticles. The nanoparticles carrying therapeutic radioisotopes should be a platform capable of not only delivering radioisotopes into the targeted tumors it should be capable of real-time imaging for the monitoring and follow-up of the treatment. In addition, the external magnetic field and other functions associated with nanoparticles can be used to enhance the delivery of radiation dosages and reduce normal tissue damage. The further development of nanoparticle-based carriers will offer a range of approaches for the targeted delivery of radioisotopes for use in the internal and systemic radiation therapy of cancers. 


\section{Acknowledgments}

Authors are grateful to Miss Tianning Fan for her assistance in illustrations and to the National Cancer Institute for their grant support of the Center of Cancer Nanotechnology Excellence (CCNE, U54 CA119338-01) and in vivo Cellular and Molecular Imaging Center (ICMIC, P50CA12830101A10003).

\section{Disclosure}

The authors report no conflicts of interest relevant to this research.

\section{References}

1. Jemal A, Siegel R, Ward E, et al. 2008. Cancer statistics. CA Cancer J Clin. 2008;58:71-96.

2. Jarzyna PA, Skajaa T, Gianella A, et al. Iron oxide core oil-in-water emulsions as a multifunctional nanoparticle platform for tumor targeting and imaging. Biomaterials. 2009;30:6947-6954.

3. Peng X, Qian X, Mao H, et al. Targeted magnetic iron oxide nanoparticles for tumor imaging and therapy. Int J Nanomed. 2008;3:311-321.

4. Yang L, Peng X, Wang A, et al. Receptor-targeted nanoparticles for in vivo imaging of breast cancer. Clin Cancer Res. 2009;15:4722-4732.

5. Shenoy D, Little S, Langer R, Amiji M. Poly(ethylene oxide)-modified poly(beta-amino ester) nanoparticles as a $\mathrm{pH}$-sensitive system for tumor-targeted delivery of hydrophobic drugs. 1. In vitro evaluations. Mol Pharm. 2005;2:357-366.

6. Bae Y, Diezi TA, Zhao A, Kwon GS. Mixed polymeric micelles for combination cancer chemotherapy through the concurrent delivery of multiple chemotherapeutic agents. J Control Release. 2007;122: 324-330.

7. Lee H, Lee E, Kim K, Jang NK, Jeong YY, Jon S. Antibiofouling polymer-coated superparamagnetic iron oxide nanoparticles as potential magnetic resonance contrast agents for in vivo cancer imaging. $J \mathrm{Am}$ Chem Soc. 2006;128:7383-7389.

8. Korb L. Radiotherapy for the palliation of prostate cancer. Semin Surg Oncol. 2000;18:75-79.

9. Hartmann F, Horak EM, Garmestani K, et al. Radioimmunotherapy of nude mice bearing a human interleukin 2 receptor alpha-expressing lymphoma utilizing the alpha-emitting radionuclide-conjugated monoclonal antibody ${ }^{212}$ Bi-anti-Tac. Cancer Res. 1994;54:4362-4370.

10. Kennel SJ, Stabin M, Roeske JC, et al. Radiotoxicity of bismuth-213 bound to membranes of monolayer and spheroid cultures of tumor cells. Radiat Res. 1999;151:244-256.

11. Ballangrud AM, Yang WH, Charlton DE, et al. Response of LNCaP spheroids after treatment with an alpha-particle emitter $\left({ }^{213} \mathrm{Bi}\right)$-labeled anti-prostate-specific membrane antigen antibody (J591). Cancer Res. 2001;61:2008-2014.

12. Humm JL. A microdosimetric model of astatine-211 labeled antibodies for radioimmunotherapy. Int J Radiat Oncol Biol Phys. 1987;13: 1767-1773.

13. Goldenberg DM. Targeted therapy of cancer with radiolabeled antibodies. J Nucl Med. 2002;43:693-713.

14. Salvatori M, Indovina1 L, Mansi L. Targeted $\alpha$-particle therapy: a clinical overview. Current Radiopharmaceuticals. 2008;1:251-253.

15. Zalutsky MR. Targeted a-particle therapy of microscopic disease: providing a further rationale for clinical investigation. $J \mathrm{Nucl} \mathrm{Med}$. 2006;47:1238-1240.

16. Mühlhausen U, Schirrmacher R, Piel M, et al. Synthesis of ${ }^{131} \mathrm{I}-$ labeled glucose-conjugated inhibitors of O6-methylguanine-DNA methyltransferase (MGMT) and comparison with nonconjugated inhibitors as potential tools for in vivo MGMT imaging. $J$ Med Chem. 2006;49:263-272.
17. Schipper ML, Riese CG, Seitz S, et al. Efficacy of $99 \mathrm{mTc}$ pertechnetate and ${ }^{131} \mathrm{I}$ radioisotope therapy in sodium/iodide symporter (NIS)-expressing neuroendocrine tumors in vivo. Eur J Nucl Med Mol Imaging. 2007;34:638-650.

18. Mulford DA, Scheinberg DA, Jurcic JG. The promise of targeted a-particle therapy. J Nucl Med. 2005;46:199S-204S.

19. Mitra A, Nan A, Line BR, et al. Nanocarriers for nuclear imaging and radiotherapy of cancer. Current Pharmaceutical Design. 2006;12:4729-4749.

20. Saravanakumar G, Kim K, Park JH, et al. Current status of nanoparticlebased imaging agents for early diagnosis of cancer and atherosclerosis. J Biomed Nanotechno. 2009;5:20-35.

21. Marcucci F, Lefoulon F. Active targeting with particulate drug carriers in tumor therapy: fundamentals and recent progress. Drug Discovery Today. 2004;9:219-228

22. Li L, Wartchow CA, Danthi SN, et al. A novel antiangiogenesis therapy using an integrin or anti-FLK-1 antibody cotated ${ }^{90}$ Y-labeled nanoparticles. Int J Radiation Oncology Biol Phys. 2004;58:1215-1227.

23. DeNardo SJ, DeNardo GL, Miers LA, et al. Development of tumor targeting bioprobes $\left({ }^{111} \mathrm{In}\right.$-chimeric L6 monoclonal antibody nanoparticles) for alternating magnetic field cancer therapy. Clin Cancer Res. 2005; 11:7087S-7092S.

24. Chen J, Wu H, Han D, et al. Using anti-VEGF McAb and magnetic nanoparticles as double-targeting vector for the radioimmunotherapy of liver cancer. Cancer Letters. 2006;231:169-175.

25. Nie S, Xing Y, Kim GJ, Simons JW. Nanotechnology applications in cancer. Annu Rev Biomed Eng. 2007;9:257-288.

26. Qian X, Peng XH, Ansari DO, et al. In vivo tumor targeting and spectroscopic detection with surface-enhanced Raman nanoparticle tags. Nat Biotechnol. 2008;26:83-90.

27. Gupta AK, Gupta M. Synthesis and surface engineering of iron oxide nanoparticles for biomedical applications. Biomaterials. 2005;26:3995-4021

28. Veiseh O, Sun C, Gunn J, et al. Optical and MRI multifunctional nanoprobe for targeting gliomas. Nano Letters. 2005;5:1003-1008.

29. Xie J, Xu C, Kohler Y, et al. Controlled PEGylation of monodisperse $\mathrm{Fe} 3 \mathrm{O} 4$ nanoparticles for reduced non-specific uptake by macrophage cells. Adv Mater. 2007;19:3163-3166.

30. Veiseh O, Sun C, Fang C, et al. Specific targeting of brain tumors with an optical/magnetic resonance imaging nanoprobe across the bloodbrain barrier. Cancer Res. 2009;69:6200-6207.

31. Sun C, Lee JS, Zhang M. Magnetic nanoparticles in MR imaging and drug delivery. Adv Drug Deliv Rev. 2008;60:1252-1265.

32. Briley-Saebo K, Bjørnerud A, Grant D, et al. Hepatic cellular distribution and degradation of iron oxide nanoparticles following single intravenous injection in rats: implications for magnetic resonance imaging. Cell Tissue Res. 2004;316:315-323.

33. Fujita T, Nishikawa M, Ohtsubo Y, et al. Control of in-vivo fate of albumin derivatives utilizing combined chemical modification. J Drug Target. 1994;2:157-165.

34. Kairdolf BA, Mancini MC, Smith AM, et al. Minimizing nonspecific cellular binding of quantum dots with hydroxyl-derivatizied surface coatings. Anal Chem. 2008;80:3029-3034.

35. Ladd J, Zhang Z, Chen S, et al. Zwitterionic polymers exhibiting high resistance to nonspecific protein adsorption from human serum and plasma. Biomacromolecules. 2008;9:1357-1361.

36. Breus VV, Heyes CD, Tron K, et al. Zwitterionic Biocompatible Quantum Dots for Wide pH Stability and Weak Nonspecific Binding to Cells. ACS Nano. 2009;3:2573-2580.

37. Yang W, Zhang L, Wang S, et al. Functionalizable and ultra stable nanoparticles coated with zwitterionic poly(carboxybetaine) in undiluted blood serum. Biomaterials. 2009;30:5617-5621.

38. Chen H, Wu X, Duan H, et al. Biocompatible polysiloxane-containing diblock copolymer PEO-b-P $\gamma$ MPS for coating magnetic nanoparticles. ACS Applied Materials and Interfaces. 2009;1:2134-2140.

39. Panyam J, Zhou WZ, Prabha S, et al. Rapid endo-lysosomal escape of poly(DL-lactide-co-glycolide) nanoparticles: implications for drug and gene delivery. FASEB J. 2002;16:1217-1226. 
40. Nasongkla N, Bey E, Ren J, et al. Multifunctional polymeric micelles as cancer-targeted, MRI-ultrasensitive drug delivery systems. Nano Letters. 2006;6:2427-2430.

41. Liu X, Wang Y, Nakamura K, et al. Auger radiation - induced, antisensemediated cytotoxicity of tumor cells using a 3-component streptavidindelivery nanoparticle with ${ }^{111} \mathrm{In} . \mathrm{J} \mathrm{Nucl} \mathrm{Med}$. 2009;50:582-590.

42. Wang MD, Shin DM, Simon JW, et al. Nanotechnology for targeted cancer therapy. Expert Rev Anticancer Ther. 2007;7:833-837.

43. Brannon-Peppas L, Blanchette JO. Nanoparticle and targeted systems for cancer therapy. Advanced Drug Delivery Reviews. 2004;56: 1649-1659.

44. van Vlerken LE, Vyas TK, Amiji MM. Poly (ethylene glycol)-modified nanocarriers for tumor-targeted and intracellular delivery. Pharmaceutical Research. 2007;24:1405-1414.

45. Kukowska-Latallo JF, Candido KA, Cao Z, et al. Nanoparticle targeting of anticancer drug improves therapeutic response in animal model of human epithelial cancer. Cancer Res. 2005;65:5317-5324.

46. Weissleder R, Kelly K, Sun EY, et al. Cell-specific targeting of nanoparticles by multivalent attachment of small molecules. Nat Biotechnol. $2005 ; 23: 1418-1423$.

47. Liu Z, Cai W, He L, et al. In vivo biodistribution and highly efficient tumour targeting of carbon nanotubes in mice. Nat Nanotechnol. 2007;2:47-52.

48. Yang L, Mao H, Wang YY, et al. Single chain epidermal growth factor receptor antibody conjugated nanoparticles for in vivo tumor targeting and imaging. Small. 2009;5:235-243.

49. Zhang CF, Jugold M, Woenne EC, et al. Specific targeting of tumor angiogenesis by RGD-conjugated ultrasmall superparamagnetic iron oxide particles using a clinical 1.5-T magnetic resonance scanner. Cancer Res. 2007;67:1555-1562.

50. Cai WB, Chen XY. Preparation of peptide-conjugated quantum dots for tumor vasculature-targeted imaging. Nature Protocols. 2008;3:89-96.

51. Antony AC. Folate receptors. Annu Rev Nutr. 1996;16:501-512.

52. Ke CY, Mathias CJ, Green MA. Folate-receptor-targeted radionuclide imaging agents. Adv Drug Deliv Rev. 2004;56:1143-1160.

53. Das M, Mishra D, Dhak P, et al. Biofunctionalized, phosphonategrafted, ultrasmall iron oxide nanoparticles for combined targeted cancer therapy and multimodal imaging. Small. 2009;5:2883-2893.

54. Thomas M, Kularatne SA, Qi L, et al. Ligand-targeted delivery of small interfering RNAs to malignant cells and tissues. Ann N Y Acad Sci. 2009;1175:32-39.

55. Setua S, Menon D, Asok A, et al. Folate receptor targeted, rare-earth oxide nanocrystals for bi-modal fluorescence and magnetic imaging of cancer cells. Biomaterials. 2010;31:714-729.

56. Rossin R, Pan D, Qi K, et al. ${ }^{64} \mathrm{Cu}$-labeled folate-conjugated shell cross-linked nanoparticles for tumor imaging and radiotherapy: synthesis, radiolabeling, and biological evaluation. J Nucl Med. 2005; 46:1210-1218.

57. Alexiou H, Jurgons R, Schmid RJ, et al. Magnetic drug targetingbiodistribution of the magnetic carrier and the chemotherapeutic agent mitoxantrone after locoregional cancer treatment. J Drug Targeting. 2003;11:139-149.
58. Raylman RR, Wahl RL. Magnetically enhanced radionuclide therapy. J Nucl Med. 1994;35:157-163.

59. Streffer C. The biological basis for tumor therapy by hyperthermia and radiation. In: Streffer J, editor. Hyperthermia and The Therapy of Malignant Tumors. Berlin: Springer; 1987.

60. Moroz P, Jones SK, Gray BN. Magnetically mediated hyperthermia: Current status and future directions. Int $J$ Hyperthermia. 2003;8:267-284.

61. Natarajan A, Xiong C, Gruettner C, et al. Development of multivalent radioimmunonanoparticles for cancer imaging and therapy. Cancer Biotherapy Radiopharmaceuticals. 2008;23:82-91.

62. Natarajan A, Gruettner C, Ivkov R, et al. Nanoferrite particle based radioimmunonanoparticles: binding affinity and in vivo pharmacokinetics. Bioconjugate Chem. 2008;19:1211-1218.

63. Sofou S. Radionuclide carriers for targeting of cancer. Int J Nanomed. 2008;3:181-199.

64. Azab AK, Srebnik M, Doviner V, et al. Targeting normal and neoplastic tissues in the rat jejunum and colon with boronated, cationic acrylamide copolymers. J Control Release. 2005;106:14-25.

65. Yinghuai Z, Peng AT, Carpenter K, et al. Substituted carboraneappended water-soluble single-wall carbon nanotubes: new approach to boron neutron capture therapy drug delivery. J Am Chem Soc. 2005; 127:9875-9880.

66. Mortensena MW, Sørensenb PG, Bjorkdahl O, et al. Preparation and characterization of Boron carbide nanoparticles for use as a novel agent in T cell-guided boron neutron capture therapy. Applied Radiation Isotopes. 2006;64:315-324.

67. Li SD, Huang L. Nanoparticles evading the reticuloendothelial system: role of the supported bilayer. Biochim Biophys Acta. 2009; 1788:2259-2266.

68. Fang C, Shi B, Pei YY, Hong MH, Wu J, Chen HZ. In vivo tumor targeting of tumor necrosis factor-alpha-loaded stealth nanoparticles: effect of MePEG molecular weight and particle size. Eur J Pharm Sci. 2006;27:27-36.

69. Wilhelm C, Billotey C, Roger J, Pons JN, Bacri JC, Gazeau F. Intracellular uptake of anionic superparamagnetic nanoparticles as a function of their surface coating. Biomaterials. 2003;24:1001-1011.

70. Lee HY, Li Z, Chen K, et al. PET/MRI dual-modality tumor imaging using arginine-gycine-aspartic (RGD) - conjugated radiolabeled iron oxide nanoparticles. J Nucl Med. 2008;49:1371-1379.

71. He X, Nie H, Wang K, Tan W, Wu X, Zhang P. In vivo study of biodistribution and urinary excretion of surface-modified silica nanoparticles. Anal Chem. 2008;80:9597-9603.

72. Nahrendorf M, Zhang H, Hembrador S, et al. Nanoparticle PET-CT imaging of macrophages in inflammatory atherosclerosis. Circulation. 2008;117:379-387.

73. Jacene HA, Filice R, Kasecamp W, et al. ${ }^{18}$ F-FDG PET/CT for monitoring the response of lymphoma to radioimmunotherapy. $\mathrm{J} \mathrm{Nucl} \mathrm{Med}$. 2009;50:8-17.

74. Esmaeli B, McLaughlin P, Pro B, et al. Prospective trial of targeted radioimmunotherapy with Y-90 ibritumomab tiuxetan (Zevalin) for front-line treatment of early-stage extranodal indolent ocular adnexal lymphoma. Ann Oncol. 2009;20:709-714.
Nanotechnology, Science and Applications

\section{Publish your work in this journal}

Nanotechnology, Science and Applications is an international, peerreviewed, open access journal that focuses on the science of nanotechnology in a wide range of industrial and academic applications. It is characterized by the rapid reporting across all sectors, including engineering, optics, bio-medicine, cosmetics, textiles, resource sustainability

\section{Dovepress}

and science. Applied research into nano-materials, particles, nanostructures and fabrication, diagnostics and analytics, drug delivery and toxicology constitute the primary direction of the journal. The manuscript management system is completely online and includes a very quick and fair peer-review system, which is all easy to use. 